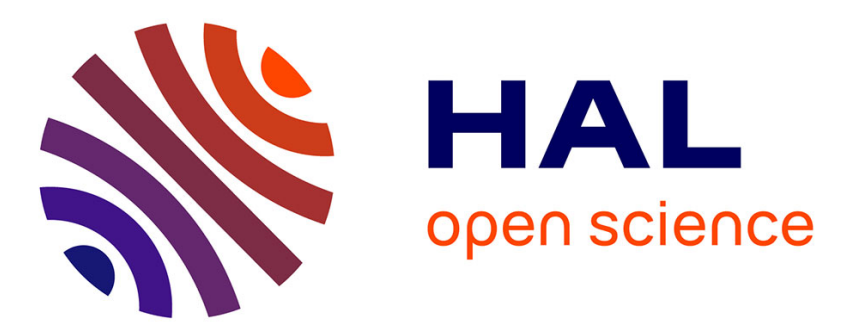

\title{
Time-Frequency Modeling and Detection of random non-stationary signals for Monitoring Purposes
}

\author{
Julien Huillery, Nadine Martin
}

\section{To cite this version:}

Julien Huillery, Nadine Martin. Time-Frequency Modeling and Detection of random non-stationary signals for Monitoring Purposes. 47th American Institut of Aeronautics and Astronautics (AIAA) conference., 2006, Newport, United States. hal-00096279

\section{HAL Id: hal-00096279 \\ https://hal.science/hal-00096279}

Submitted on 19 Sep 2006

HAL is a multi-disciplinary open access archive for the deposit and dissemination of scientific research documents, whether they are published or not. The documents may come from teaching and research institutions in France or abroad, or from public or private research centers.
L'archive ouverte pluridisciplinaire HAL, est destinée au dépôt et à la diffusion de documents scientifiques de niveau recherche, publiés ou non, émanant des établissements d'enseignement et de recherche français ou étrangers, des laboratoires publics ou privés. 


\title{
Time-Frequency Modeling and Detection of random non-stationary signals for Monitoring Purposes
}

\author{
Julien HUILLERY* and Nadine MARTIN ${ }^{\dagger}$ \\ Signal and Image Laboratory (LIS), 38402 St Martin d'Heres, France
}

\begin{abstract}
This paper deals with the modelization and detection of non-stationary random signals in the time-frequency space. A time-frequency random model of signal is derived from a given temporal model. The time model we are interested in consists in a deterministic signal embedded in an additive centered Gaussian perturbation. This Gaussian model is characterized by two parameters, which are the mean and covariance matrix of the process. The corresponding time-frequency model depends on the time-frequency transform applied to the signal. For the spectrogram, the determinant parameters are the nature and length of the analysis window and the zero-padding. We show that for a Gaussian signal, spectrogram coefficients distribution can be approximated by a $c h i^{2}$ law defined by three parameters. A time-frequency signal detection task inspired from a Neyman-Pearson strategy is performed on the basis of this probabilistic time-frequency model. The detector determines the time-frequency regions where signal energy is present. It thus provides a time-frequency signature of the signal. This information is used for structural health monitoring techniques. Extraction of the fundamental meshing frequency and harmonics of a gearbox under varying load conditions is presented.
\end{abstract}

\section{Introduction}

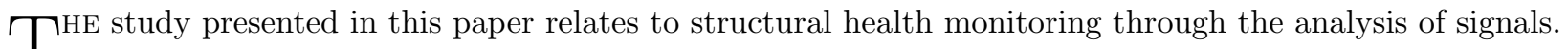
1 As monitoring is linked with the observation of the behaviour of a system, it reveals an intrinsically nonstationary context. Time-Frequency (TF) research aims at the development of new analysis tools dedicated to non-stationary signals. These tools have proved great efficiency for signal analysis as they allow the observation of the evolution along time of the signal frequency structure.

A large amount of work has been done in the field of analysis of random signals, based on time series and/or spectral analysis where effective tools constructed on a theoretical framework have been developed for detection, estimation and classification. An essential aspect of probabilistic approaches for signal analysis is the choice of the structure and nature of the model used to represent the randomness of the signal. This modelization always concerns the time representation of the signal. The first aim of this paper is to present how a time random model can be transplanted into the time-frequency domain.

Similarly to the time domain, this probabilistic time-frequency model can be used as a framework for a time-frequency signal detection task. Time-frequency detection extract the time-frequency locations where a signal is present. It reveals useful for the determination of the signal temporal locations but also provides deeper information about the spectral structure of the signal and how this structure evolves. In this paper we present a procedure, based upon a Neyman-Pearson framework, that localizes the regions of the timefrequency plan where signal energy is present.

Structural health monitoring task based upon this analysis of the signal is performed as described in figure 1. We investigate vibration monitoring of a rotative machinery under fluctuating load conditions. Different loads imposed to an induction motor cause the rotative speed to fluctuate. Our aim is to recover the law of the load by extracting the fundamental meshing frequency of the system. The time-frequency detection procedure is used for this purpose.

\footnotetext{
*Phd student, LIS, julien.huillery@lis.inpg.fr

${ }^{\dagger}$ Research Director CNRS, LIS, nadine.martin@lis.inpg.fr
} 


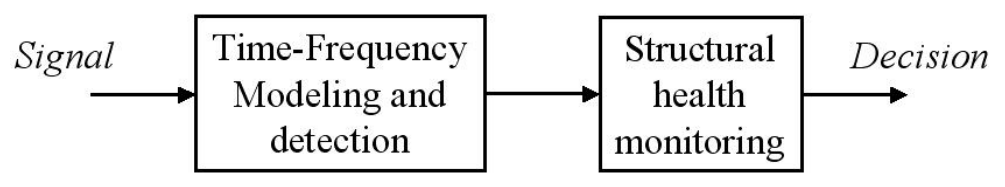

Figure 1. Overview of the method

This paper is organised as follow: the first section describes the time-frequency model of signal and the second section presents the algorithm used to detect the signal time-frequency patterns. The third part focuses on the use of the time-frequency signal detection algorithm for load variations monitoring purpose.

\section{Time-frequency modeling}

The time random model of signal we are interested in is composed of a deterministic part $d(t)$ and an additive random part $r(t)$, namely

$$
x(t)=d(t)+r(t) .
$$

The deterministic part represents the support of the information under interest while the random part stands for the receiver environment. The non-stationarities originate from the deterministic process. The structure of this model allows the development of signal processing methods without prior hypotheses upon the nature or structure of the information contained in the signal. The only a priori information is the random nature of the additive perturbation process $r(t)$. It namely consists in its law of probability that can be represented by its probability density function or equivalent. The results are here presented for a centered Gaussian process with covariance matrix $\mathbf{R}$ associated to the autocorrelation function $\Gamma(\tau)$. As a consequence, each sample $x(t)$ of the signal is distributed as a Gaussian variable with mean $d(t)$ and autocorrelation function $\Gamma(\tau)$

$$
x(t) \sim \mathcal{N}(d(t), \Gamma(\tau)) .
$$

Once the time modelization task of the phenomenon under investigation has been performed, the next step consists in determining the corresponding random model in the time-frequency domain.

Many time-frequency representations of signals have been proposed yielding to the need of a classification. ${ }^{1}$ One first distinction separates representations associated with linear or quadratic transformation of the signal. Short-Time Fourier Transform and Wavelet Transform are members of the linear group. The advantage of the quadratic group is to offer the possibility of an interpretation of time-frequency representations as physical quantities like energy. The class of Cohen gathers some of the quadratic transforms. One key aspect regarding the choice of a quadratic transform is the balance between energy concentration and presence of interference terms. The Wigner-Ville distribution (presence of interferences, high energy concentration) and the spectrogram (no interferences, poor energy concentration) correspond to the farthest transforms. To each time-frequency representation of the signal will correspond a specific time-frequency random model. The law of probability of the time-frequency coefficients will depend on the transformation applied to the signal. In this study, results are presented for the case of spectrogram.

Given a discrete analysis window $w[n]$ of length $M$, the Short-Time Fourier Transform $X_{w}[n, k]$ of a discrete signal $x[n]$ is formed by the successive Discrete Fourier Transforms of the windowed signal. The spectrogram $S_{x}^{w}[n, k]$ corresponds to the squared modulus of the STFT or equivalently to the sum of the squares of the STFT real and imaginary parts, $X_{w}^{r}[n, k]$ and $X_{w}^{i}[n, k]$ respectively. We thus start from the following definitions:

$$
\begin{gathered}
S_{x}^{w}[n, k]=X_{w}^{r}[n, k]^{2}+X_{w}^{i}[n, k]^{2}, \\
X_{w}^{r}[n, k]=\sum_{m=0}^{M-1} x[n-m] w[m] \cos \left(-2 \pi k \frac{m}{N}\right), \\
\text { American Institute of Aeronautics and Astronautics }
\end{gathered}
$$




$$
X_{w}^{i}[n, k]=\sum_{m=0}^{M-1} x[n-m] w[m] \sin \left(-2 \pi k \frac{m}{N}\right) .
$$

where $N$ is the length of the computed FFT and $N-M$ corresponds to the zero-padding.

The law of the random variable $S_{x}^{w}[n, k]$ is thus determined by the probabilistic behaviour of the squared modulus of the vector $\mathbf{X}[n, k]$ defined by

$$
\mathbf{X}[n, k]^{T}=\left[X_{w}^{r}[n, k] X_{w}^{i}[n, k]\right] .
$$

Equations (4) and (5) express the real and imaginary parts of the STFT as linear combinations of signal samples. As $x[n]$ are Gaussian variables (not necessarily independent), $X_{w}^{r}[n, k]$ and $X_{w}^{i}[n, k]$ are also distributed as Gaussian laws. $\mathbf{X}[n, k]$ is thus a Gaussian vector defined by five parameters $m_{1}, m_{2}, \Sigma_{11}$, $\Sigma_{22}$ and $\Sigma_{12}$ (for clarity of notation we drop the time-frequency location $[n, k]$ in the probability parameter notations):

$$
\mathbf{X}[n, k]=\left(\begin{array}{c}
X_{w}^{r}[n, k] \\
X_{w}^{i}[n, k]
\end{array}\right) \sim \mathcal{N}\left\{\left(\begin{array}{c}
m_{1} \\
m_{2}
\end{array}\right),\left(\begin{array}{cc}
\Sigma_{11} & \Sigma_{12} \\
\Sigma_{12} & \Sigma_{22}
\end{array}\right)\right\} .
$$

From equations (1),(4) and (5), we obtain

$$
\begin{aligned}
& m_{1}=D_{w}^{r}=\operatorname{Re}(\operatorname{STFT}\{d(t)\}) \\
& m_{2}=D_{w}^{i}=\operatorname{Im}(\operatorname{STFT}\{d(t)\})
\end{aligned}
$$

and

$$
\begin{aligned}
& \Sigma_{11}=W^{T} C_{k} \mathbf{R} C_{k} W \\
& \Sigma_{22}=W^{T} S_{k} \mathbf{R} S_{k} W \\
& \Sigma_{12}=W^{T} C_{k} \mathbf{R} S_{k} W
\end{aligned}
$$

where $W^{T}=[w[0] \cdots w[M-1]]$ is the analysis window vector and $C_{k}$ (resp. $S_{k}$ ) is the cosine (resp. sine) diagonal matrix,

$$
\begin{aligned}
& C_{k}=\operatorname{diag}\left[\cos \left(-2 \pi k \frac{m}{N}\right)\right]_{m=0, M-1} \\
& S_{k}=\operatorname{diag}\left[\sin \left(-2 \pi k \frac{m}{N}\right)\right]_{m=0, M-1}
\end{aligned}
$$

Spectrogram coefficients are thus distributed as the squared modulus of the Gaussian vector $\mathbf{X}$. This law of probability is determined through the five parameters $m_{1}, m_{2}, \Sigma_{11}, \Sigma_{22}$ and $\Sigma_{12}$. This distribution can be approximated $^{2}$ with a $c h i^{2}$ distribution with three parameters $\chi^{2}(\alpha, \delta, \theta)$. The coefficients of proportionality $\alpha$, the degree of freedom $\delta$ and the uncentrality parameter $\theta$ are then defined as

$$
\begin{gathered}
\delta=\frac{\left(\Sigma_{11}+\Sigma_{22}\right)^{2}}{\Sigma_{11}^{2}+\Sigma_{22}^{2}+2 \Sigma_{12}^{2}}, \\
\alpha=\frac{\Sigma_{11}+\Sigma_{22}}{\delta} \\
\theta=\frac{m_{1}^{2}+m_{2}^{2}}{\alpha}=\frac{S_{d}^{w}}{\alpha}
\end{gathered}
$$

where $S_{d}^{w}$ is the spectrogram of the deterministic part $d(t)$ alone.

One advantage of this time-frequency random model is the direct link between the transform that is used and the resulting distribution. Parameters of the time-frequency transformation being known, the parameters of the time-frequency model are defined by the time-frequency distribution $D_{w}[n, k]$ of the deterministic part and the variance-covariance matrix $\mathbf{R}$ of the additive random process. 


\section{Time-frequency signal detection}

We use the results presented in the previous section to develop an algorithm that automatically detect and isolate time-frequency components of the determinist part of our model. In this work, a time-frequency component of a signal is defined as a region of the time-frequency plane where signal energy is present. Furthermore, the additive random process $r(t)$ is assumed to be white with an unkown spectral power $\sigma^{2}$. We can thus write $\mathbf{R}=\sigma^{2} \mathbf{I}_{N}$. For this simple case, the three parameters $(\alpha, \delta, \theta)$ of the $\chi^{2}$ law (equations 13,14 and 15) reduce to

$$
\begin{gathered}
\delta=\frac{1}{2 \beta^{2}-2 \beta+1}, \\
\alpha=\frac{\sigma^{2}}{\delta}, \\
\theta=\frac{m_{1}^{2}+m_{2}^{2}}{\alpha}=\frac{S_{d}^{w}}{\alpha},
\end{gathered}
$$

where $\beta$ is a function of the analysis window $w[n]$ and the frequency $k$ defined by

$$
\beta=\frac{1}{2}+\frac{1}{2} * \sum_{n=0}^{M-1} w[n]^{2} \cos \left(4 \pi k \frac{n}{N}\right) .
$$

Note that for most frequencies $k, \beta$ equals $\frac{1}{2}$ and the $\chi^{2}$ law has two degrees of freedom $(\delta=2) .^{3}$

At each time-frequency location $[n, k]$, the detection scheme can be formulated as a two hypothesis test problem. The null hypothesis $H_{0}$ corresponds to the presence of the random additive process $r(t)$ only, and the signal hypothese $H_{1}$ is valid if the deterministic part $d(t)$ provides energy at the considered location. That is:

$$
\left\{\begin{array}{l}
H_{0}: S_{x}^{w}[n, k]=S_{r}^{w}[n, k] \\
H_{1}: S_{x}^{w}[n, k]=S_{r+d}^{w}[n, k]
\end{array}\right.
$$

From section II and above, the distribution of $S_{x}^{w}[n, k]$ under $H_{0}$ is a central $(\theta=0) \chi^{2}$ law for every time-frequency location $[n, k]$. The proportionality coefficient $\alpha$ is equal to $\sigma^{2} / \delta$ where the degree of freedom $\delta$ and the spectral power $\sigma^{2}$ remains to be estimated. Under $H_{1}$, spectrogram coefficients are distributed as a non-central $\chi^{2}$ variable. As for this case the non-centrality parameter $\theta$ is fluctuating over the timefrequency plane, a Neyman-Pearson strategy seems preferable for the detection task: a given fixed False Alarm Probability (PFA) determines the detection threshold on the probability distribution under $H_{0}$.

The general principle of the algorithm ${ }^{4}$ is to iteratively select higher energy connected time-frequency coefficients until the distribution of the remaining region matches a possible $H_{0}$ distribution. Doing so the algorithm iteratively estimates the unknown parameter $\sigma^{2}$ of the random process.

The time-frequency detection procedure is now described:

In the time frequency plane, candidates to the signal class $H_{1}$ are selected through the use of a PFA on the probability distribution of the noise class $H_{0}$. For this purpose, parameters $\alpha$ and $\delta$ (proportionality coefficient and degree of freedom respectively) of the central $\chi^{2}$ law are estimated ${ }^{5}$ by maximising the likelihood with the data contained in the noise class $H_{0}$. A threshold is determined on this probability distribution according to the initialy given PFA. TF coefficients $S_{x}^{w}[n, k]$ superior to this threshold are selected as candidates for the signal class $H_{1}$.

The signal class regions then grow by clustering candidates connected in the time-frequency plane. A candidate for the signal class is assigned to the class $H_{1}$ if it is contiguous to a member of the class $H_{1}$. The time-frequency region growing stops when the number of candidates falls under a threshold determined with the initial number of candidates and the PFA. 
After each grow, the likelihood of the remaining noise class distribution with a central $\chi^{2}$ distribution is evaluated. The procedure repeats iteratively until the convergence of the likelihood stops its evolution.

In fine, the limit between the noise and signal regions is determined according to the time-frequency model. The spectral power $\sigma^{2}$ of the additive random process is estimated. The time-frequency space is segmented into regions corresponding to the deterministic signal components and may be interpreted as a time-frequency signature.

\section{Load variations monitoring}

We used the time-frequency detection procedure described in section III for the analysis of vibration signals from rotative machinery. The aim of the experiment is the monitoring of a fluctuating load applied to the motor. Excess load may then be detected so as to prevent mechanical failure or motor break down.

The experiment has been carried out using data from the benchmark "GOTIX" (see figure 2) of the Signal and Image Laboratory, Grenoble, France. This benchmark is composed of a $55 \mathrm{~kW}$ asynchronous motor, a gearbox with a multiplicative ratio of $57 / 15$, a speed variable controller and a DC motor that allows the simulation of variable loads. Signals are acquired from various voltage/current sensors and accelerometers placed at different locations. We used the vibration signal recorded on the motor shaft. During acquisition, the load represented by the DC motor was continuously modified.

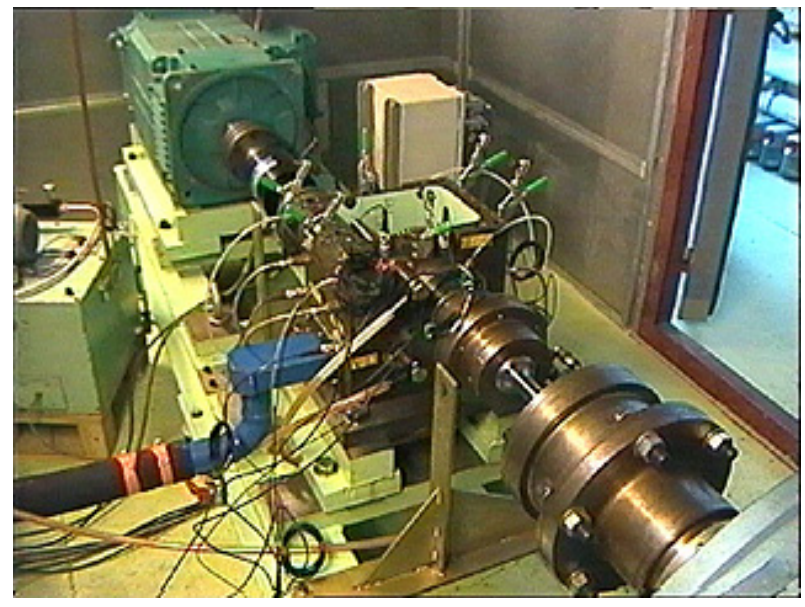

(a) global view

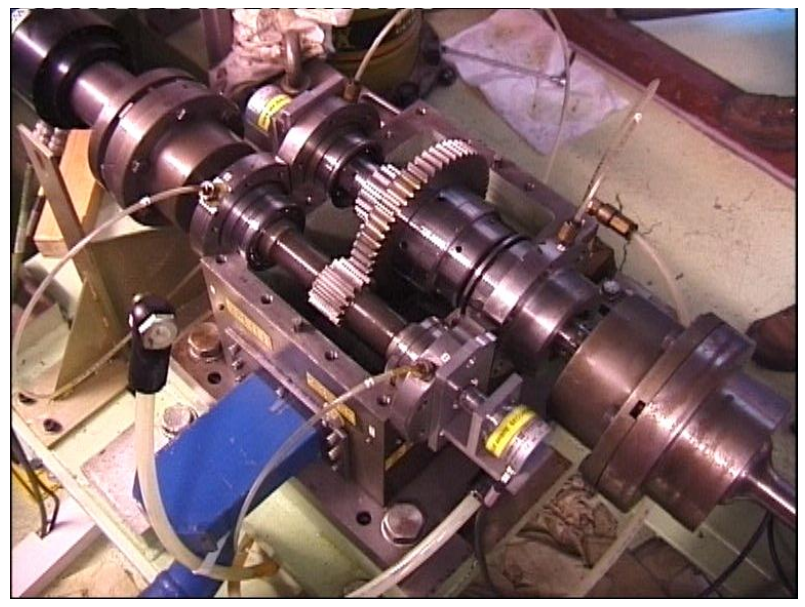

(b) detail of the gearbox

Figure 2. The benchmark "GOTIX" from the Signal and Image Laboratory (LIS)

Vibration signals from rotative machinery display a harmonic structure (see figure 3-a)). The fundamental frequency corresponds to the rotation speed of the motor. Variation of the load imposed to the rotative machinery results in inversely proportional variation of the rotation speed. As a consequence, extraction of the fundamental meshing frequency of the vibration signal allows to recover the load conditions the system has been subject to.

Unfortunately, the fundamental frequency of vibration signals may have less energy than some of its harmonics and suffer from amplitude variations. Furthermore, a load variation $\Delta L$ generates a frequency variation of $K \Delta L$ on the fundamental frequency and $n * K \Delta L$ on the $n^{t h}$ harmonic. As the frequency resolution of a time-frequency analysis is constant over the whole frequency range, resolution on the load variation is gained when looking at high harmonics. In the case under interest, the $7^{\text {th }}$ harmonic reveals the more energetic and of relatively stable amplitude (figure 3-b)). We thus restrict the analysis to the frequency band $[5200 \mathrm{~Hz} ; 6100 \mathrm{~Hz}]$. Some knowledge about the behaviour of the investigated rotative machinery and sensors may help to determine this spectral region of interest as it remained the same for all the experiments we conducted.

Figure 3-c) depicts the time-frequency signal detection result from which the load variations are extracted (figure 3-d)) by use of the motor torque/rotation speed characteristic. We recover the load variations manually obtained during the experiment. 


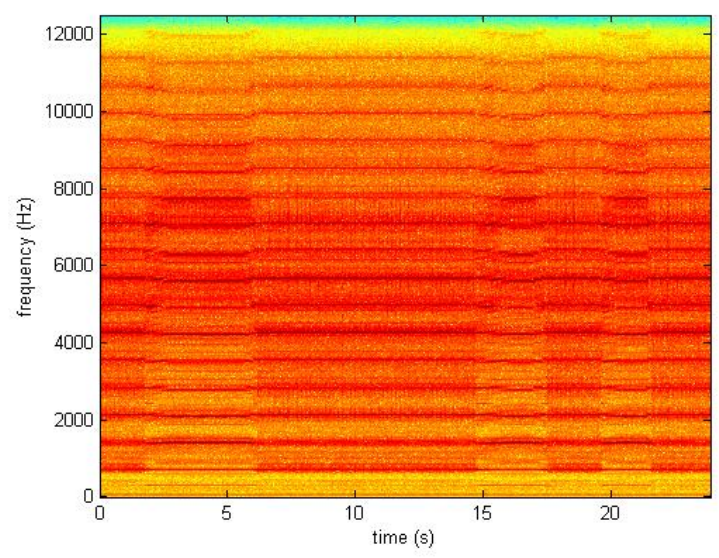

(a) spectrogram of the gear signal

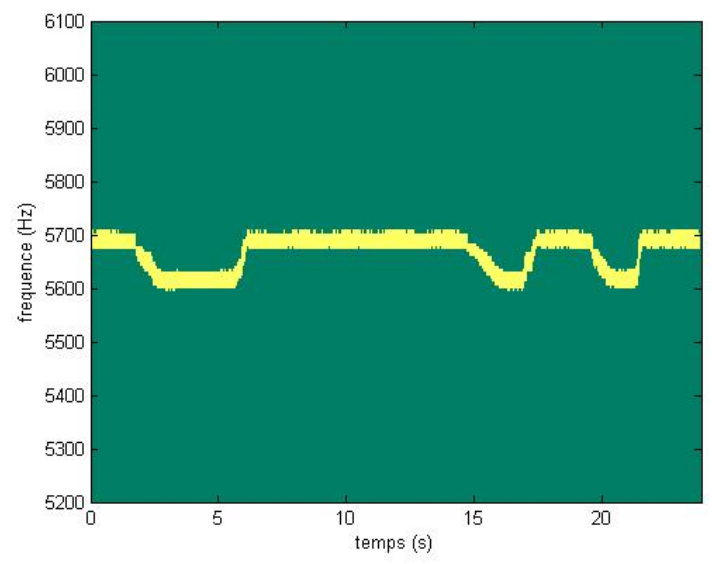

(c) Time-frequency detection result

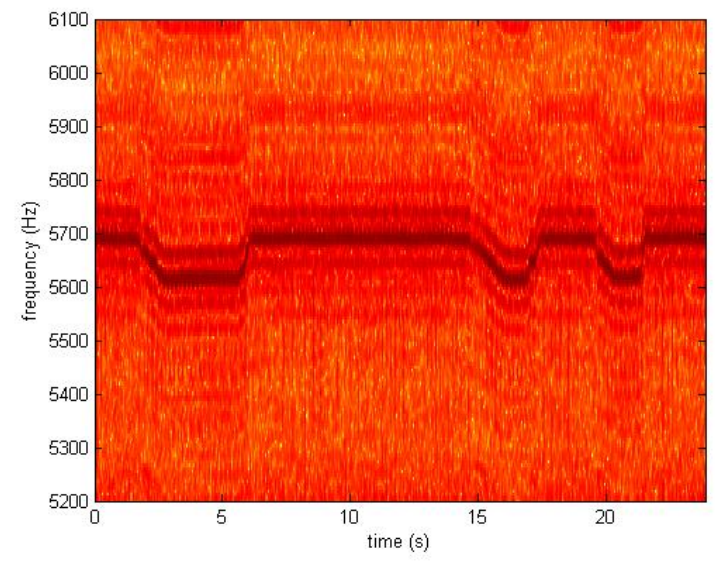

(b) zoom on the $7^{\text {th }}$ harmonic

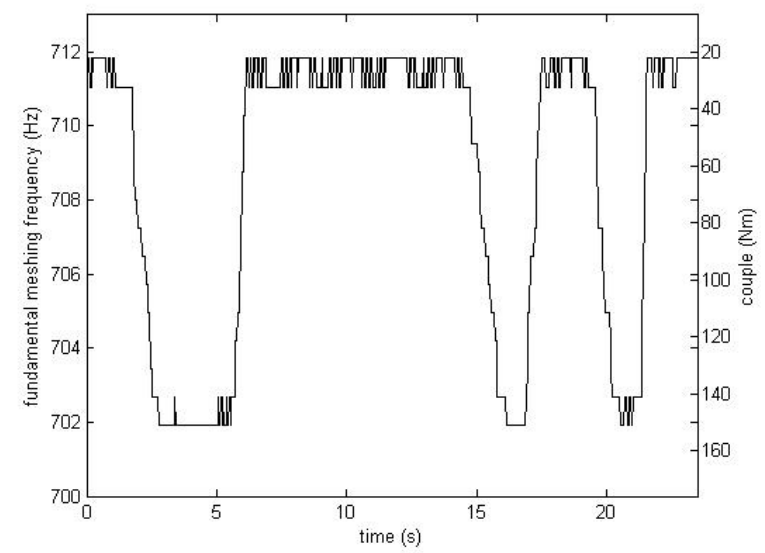

(d) fundamental meshing frequency variations (left y-axis) and corresponding load conditions (rigth y-axis)

Figure 3. Time-frequency signal detection and load variations monitoring

\section{Conclusion}

More than extract the temporal location of a signal, time-frequency signal detection provides information about the spectral structure of a signal and the way it evolves. In this study, a time-frequency detection procedure is presented. This procedure is based on a time-frequency probabilistic model constructed on a given Gaussian temporal model and taking into account the characteristics of the time-frequency transform used for the analysis. Monitoring of the load imposed to a rotative machinery is presented and show the applicability of the method.

\section{References}

${ }^{1}$ Cohen, L., "Time-Frequency Distribution - A Review," Proceedings of the IEEE, Vol. 77, No. 7, July 1989, pp. $941-981$.

${ }^{2}$ Huillery, J., Millioz, F., and Martin, N., "On the Probability Distributions of Spectrogram coefficients for Correlated Gaussian Process," In proceedings of the ICASSP'06, Toulouse, France, May 2006.

${ }^{3}$ Millioz, F., Huillery, J., and Martin, N., "Short-Time Fourrier Transform Probability Distribution for Time-Frequency Segmentation," In proceedings of the ICASSP'06, Toulouse, France, May 2006.

${ }^{4}$ Hory, C., Martin, N., and Chehikian, A., "Spectrogram Segmentation by means of Statistical Features for Non-stationary Signal Interpretation," IEEE Trans. on Signal Processing, Vol. 50, No. 12, December 2002, pp. 2915-2925.

${ }^{5}$ Hory, C. and Martin, N., "Maximum likelihood noise estimation for spectrogram segmentation control," In Proceedings of the ICASSP'02, Orlando, USA, 2002, pp. 1581-1584. 